\title{
THE SYZ MIRROR SYMMETRY AND THE BKMP REMODELING CONJECTURE
}

\author{
BOHAN FANG, CHIU-CHU MELISSA LIU, AND ZHENGYU ZONG
}

\begin{abstract}
The Remodeling Conjecture proposed by Bouchard-Klemm-MariñoPasquetti (BKMP) relates the A-model open and closed topological string amplitudes (open and closed Gromov-Witten invariants) of a symplectic toric Calabi-Yau 3-fold to Eynard-Orantin invariants of its mirror curve. The Remodeling Conjecture can be viewed as a version of all genus open-closed mirror symmetry. The SYZ conjecture explains mirror symmetry as $T$-duality. After a brief review on SYZ mirror symmetry and mirrors of symplectic toric Calabi-Yau 3-orbifolds, we give a non-technical exposition of our results on the Remodeling Conjecture for symplectic toric Calabi-Yau 3-orbifolds. In the end, we apply SYZ mirror symmetry to obtain the descendent version of the all genus mirror symmetry for toric Calabi-Yau 3-orbifolds.
\end{abstract}

\section{INTRODUCTION}

1.1. The SYZ conjecture. Mirror symmetry relates the A-model on a Calabi-Yau $n$-fold $\mathcal{X}$, defined by the symplectic structure on $\mathcal{X}$, to the B-model on a mirror Calabi-Yau $n$-fold $\check{X}$, defined by the complex structure on $\check{X}$. Strominger-YauZaslow proposed that Mirror Symmetry is T-duality [110 in the following sense. There exist $\pi: \mathcal{X} \rightarrow B$ and $\check{\pi}: \check{\mathcal{X}} \rightarrow B$, where $\operatorname{dim}_{\mathbb{R}} B=n$, such that over a dense open subset $U \subset B, Y:=\pi^{-1}(U) \rightarrow U$ and $\check{Y}:=\check{\pi}^{-1}(U) \rightarrow U$ are dual special Lagrangian $n$-torus fibrations. In the semi-flat case, $U=B, Y \cong T^{*} B / \Lambda$ as a symplectic manifold and $\check{Y} \cong T B / \Lambda^{\vee}$ as a complex manifold, where $\Lambda$ and $\Lambda^{\vee}$ are dual lattices, and for each $b \in B, \pi^{-1}(b)=T_{b}^{*} B / \Lambda$ and $\check{\pi}^{-1}(b)=T_{b} B / \Lambda^{\vee}$. In the general case, there is quantum correction to the complex structure on $\check{Y}$ coming from holomorphic disks in $\mathcal{X}$ bounded by Lagrangian $n$-tori $\pi^{-1}(b), b \in U$.

Mirror symmetry has been extended to certain non-Calabi-Yau manifolds. When $\mathcal{X}$ is Fano (or more generally semi-Fano), the mirror is a Landau-Ginzburg model, which can also be constructed by SYZ transformation.

1.2. SYZ mirror symmetry for toric Calabi-Yau 3 -folds. Let $(\mathcal{X}, \omega)$ be a symplectic toric Calabi-Yau 3-manifold/orbifold, where $\omega$ is the symplectic form. There are two families of mirrors, both of which can be constructed by the SYZ transformation.

Landau-Ginzburg mirror. The mirror B-model to the toric Calabi-Yau 3-orbifold $(\mathcal{X}, \omega)$ is a 3 -dimensional Landau-Ginzburg model on $\left(\mathbb{C}^{*}\right)^{3}$ given by the superpotential

$$
W=H(X, Y, q) Z .
$$

The Calabi-Yau condition ensures that $W$ is in this form. The complex parameter $q$ is related to the Kähler parameter of $\mathcal{X}$ by the mirror map. The Landau-Ginzburg mirror $\left(\left(\mathbb{C}^{*}\right)^{3}, W\right)$ can be constructed by applying SYZ transformation to $\mu: \mathcal{X} \rightarrow$ 
$\Delta \subset \mathbb{R}^{3}$, where $\mu$ is the moment map of the Hamiltonian $U(1)^{3}$-action on $(\mathcal{X}, \omega)$, and $\Delta=\mu(\mathcal{X})$ is the moment polyhedron.

Hori-Vafa mirror. By Hori-Vafa [66, the mirror of $(\mathcal{X}, \Omega)$ is a non-compact CalabiYau 3-fold $(\check{\mathcal{X}}, \Omega)$, where

$$
\check{\mathcal{X}}=\left\{(u, v, X, Y): u, v \in \mathbb{C}, X, Y \in \mathbb{C}^{*}, u v=H(X, Y)\right\}
$$

is a hypersurface in $\mathbb{C}^{2} \times\left(\mathbb{C}^{*}\right)^{2}$, and

$$
\Omega=\operatorname{Res}_{\check{\mathcal{X}}}\left(\frac{1}{u v-H(X, Y)} d u \wedge d v \wedge \frac{d X}{X} \wedge \frac{d Y}{Y}\right)
$$

is a holomorphic 3 -form on $\check{\mathcal{X}}$. The Hori-Vafa mirror $(\check{\mathcal{X}}, \Omega)$ can be constructed by applying SYZ transformation to the Gross fibration [27, 26].

The two "equivalent" mirrors come with no surprise since as a toric variety $\mathcal{X}$ should have a Landau-Ginzburg mirror, while as a Calabi-Yau 3-fold Hori-Vafa showed that they both could be reduced to a mirror curve

$$
C_{q}=\left\{(X, Y) \in\left(\mathbb{C}^{*}\right)^{2}: H(X, Y, q)=0\right\} \subset\left(\mathbb{C}^{*}\right)^{2} .
$$

In particular

$$
\int_{\Gamma} e^{-W} \frac{d X d Y d Z}{X Y Z}=\int_{\widetilde{\Gamma}} \Omega=\int_{\gamma} y d x
$$

where $x=-\log X, y=-\log Y$, and Lagrangian cycles $\Gamma \subset\left(\mathbb{C}^{*}\right)^{3}, \widetilde{\Gamma} \subset \check{\mathcal{X}}, \gamma \subset C_{q}$ are related by a series of dimensional reductions.

1.3. The BKMP remodeling conjecture. It is usually difficult to obtain higher genus invariants of the B-model. A standard way is to apply the BCOV holomorphic anomaly equations [15. These equations do not have unique solutions (holomorphic ambiguity). One needs to fix the boundary conditions via the input from mirror symmetry, like Gromov-Witten invariants in low degrees in the large radius limit point, and to utilize the so-called "gap conditions" at the conifold point. To mathematically prove the all genus mirror symmetry under this B-model approach is currently beyond reach since the A-side theory of BCOV (and the A-side theory at the conifold point) is still a mystery to mathematicians.

The Eynard-Orantin topological recursion is an algorithm which produces higher genus invariants for a spectral curve [46. Applying the Eynard-Orantin topological recursion to the mirror curve $C_{q}$ of a symplectic toric Calabi-Yau 3-orbifold $(\mathcal{X}, \omega)$, we obtain a version of the B-model, related but not a priori the same as the BCOV theory on the Hori-Vafa mirror $(\dot{\mathcal{X}}, \Omega)$ of $(\mathcal{X}, \omega)$. The Bouchard-Klemm-MariñoPasquetti (BKMP) remodeling conjecture 20, 21] says that all genus B-model topological strings on $(\check{\mathcal{X}}, \Omega)$ are essentially Eynard-Orantin invariants [46] of $C_{q}$. Using mirror symmetry, BKMP relates the Eynard-Orantin invariant $\omega_{g, n}$ of the mirror curve to a generating function $F_{g, n}^{\mathcal{X}, \mathcal{L}}$ of open $\mathrm{GW}$ invariants (A-model topological open string amplitudes) counting holomorphic maps from bordered Riemann surfaces with $g$ handles and $n$ holes to $\mathcal{X}$ with boundaries in an Aganagic-Vafa Lagrangian brane $\mathcal{L}$. The correspondence between $\omega_{g, n}$ and $F_{g, n}^{\mathcal{X}, \mathcal{L}}$ can be extend to the case when $n=0$. In this case, $\omega_{g, 0}$ is defined to be the free energy and the Amodel potential becomes the closed Gromov-Witten potential $F_{g}^{\mathcal{X}}$. Therefore, the remodeling conjecture gives us an all genus open-closed mirror symmetry for toric 
Calabi-Yau 3-orbifolds. The three equivalent B-models mirror to $\mathcal{X}$ are illustrated as below.

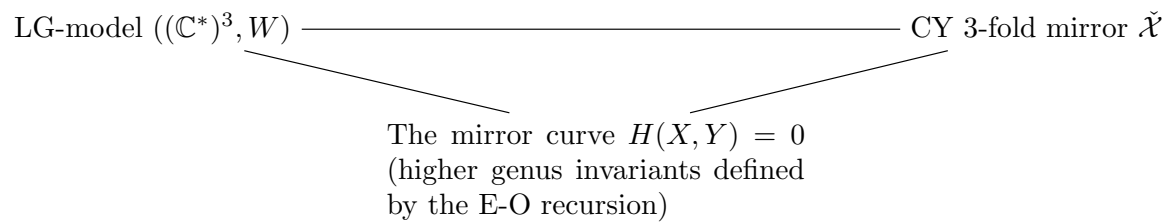

The BKMP remodeling conjecture was proved for $\mathbb{C}^{3}$ at all genus $g$ independently by L. Chen [28] and J. Zhou [116] in the $n>0$ case (open string sector), and by Bouchard-Catuneanu-Marchal-Sułkowski [19] in the $n=0$ case (closed string sector). In 2012, Eynard-Orantin provided a proof of the BKMP remodeling conjecture for all symplectic smooth toric Calabi-Yau 3-folds [47.

The SYZ T-duality transformation [110, 9, 78, associates a coherent sheaf $\mathcal{F}$ on $\mathcal{X}$ to a Lagrangian cycle $\operatorname{SYZ}(\mathcal{F}) \subset\left(\mathbb{C}^{*}\right)^{3}$. A coherent sheaf on $\mathcal{X}$ is admissible if there exists $\gamma(\mathcal{F}) \subset C_{q}$ such that

$$
\int_{\mathrm{SYZ}(\mathcal{F})} e^{\frac{W+x+f y}{z}} \frac{d X d Y d Z}{X Y Z}=\int_{\gamma(\mathcal{F})} e^{\frac{x+f y}{z}} y d x
$$

and $x+f y$ is bounded below on $\gamma(\mathcal{F})$. Here $f$ is an integer and $z$ is a negative real number, so that the integral on the right hand side converges. The remodeling conjecture has a descendant version: given $n$ admissible coherent sheaves $\mathcal{F}_{1}, \ldots, \mathcal{F}_{n}$ on $\mathcal{X}$, the Laplace transform of $\omega_{g, n}$ along $\gamma\left(\mathcal{F}_{1}\right), \ldots, \gamma\left(\mathcal{F}_{n}\right)$ is a generating function of genus $g$ descendant Gromov-Witten invariants with $n$ insertions $\kappa\left(\mathcal{F}_{1}\right), \ldots, \kappa\left(\mathcal{F}_{n}\right)$, where $\kappa\left(\mathcal{F}_{i}\right)$ is the so-called Gamma class of $\mathcal{F}_{i}$.

In the rest of this paper, we will give a non-technical exposition of our results on the remodeling conjecture for toric Calabi-Yau 3-orbifolds [51, 52, which is a version of all genus open-closed mirror symmetry. We will also discuss the all genus mirror symmetry of free energies and descendant potentials.

\section{Toric Calabi-Yau 3-Orbifolds and their mirror CURVES}

2.1. Toric Calabi-Yau 3-orbifolds. A Calabi-Yau 3-fold $X$ is toric if it contains the algebraic torus $\mathbb{T}=\left(\mathbb{C}^{*}\right)^{3}$ as a Zariski dense open subset, and the action of $\mathbb{T}$ on itself extends to $X$. All Calabi-Yau 3-folds are non-compact. There is a rank 2 subtorus $\mathbb{T}^{\prime} \subset \mathbb{T}$ which acts trivially on the canonical line bundle of $X$. We call $\mathbb{T}^{\prime}$ the Calabi-Yau torus. Then $\mathbb{T} \cong \mathbb{T}^{\prime} \times \mathbb{C}^{*}$. Let $\mathbb{T}_{\mathbb{R}}^{\prime} \cong U(1)^{2}$ be the maximal compact subgroup of $\mathbb{T}^{\prime}$.

Let $M^{\prime}=\operatorname{Hom}\left(\mathbb{T}^{\prime}, \mathbb{C}^{*}\right) \cong \mathbb{Z}^{2}$ and $N^{\prime}=\operatorname{Hom}\left(\mathbb{C}^{*}, \mathbb{T}^{\prime}\right)$ be the character lattice and the cocharacter lattice of $\mathbb{T}^{\prime}$, respectively. Then $M^{\prime}$ and $N^{\prime}$ are dual lattices. Let $X_{\Sigma}$ be a toric Calabi-Yau 3-fold defined by a simplicial fan $\Sigma \subset N_{\mathbb{R}}^{\prime} \times \mathbb{R}$, where $N_{\mathbb{R}}^{\prime}:=N^{\prime} \otimes_{\mathbb{Z}} \mathbb{R} \cong \mathbb{R}^{2}$ can be identified with the Lie algebra of $\mathbb{T}_{\mathbb{R}}^{\prime}$. Then $X_{\Sigma}$ has at most quotient singularities. We assume that $X_{\Sigma}$ is semi-projective, i.e., $X_{\Sigma}$ contains at least one $\mathbb{T}$ fixed point, and $X_{\Sigma}$ is projective over its affinization $X_{0}:=\operatorname{Spec} H^{0}\left(X_{\Sigma}, \mathcal{O}_{X_{\Sigma}}\right)$. Then the support of the fan $\Sigma$ is a strongly convex rational polyhedral cone $\sigma_{0} \subset N_{\mathbb{R}}^{\prime} \times \mathbb{R} \cong \mathbb{R}^{3}$, and $X_{0}$ is the affine toric variety defined by the 3 -dimensional cone $\sigma_{0}$. There exists a convex polytope $P \subset N_{\mathbb{R}}^{\prime} \cong \mathbb{R}^{2}$ with vertices in the lattice $N^{\prime} \cong \mathbb{Z}^{2}$, such that $\sigma_{0}$ is the cone over $P \times\{1\} \subset N_{\mathbb{R}}^{\prime} \times \mathbb{R}$, i.e. $\sigma_{0}=\{(t x, t y, t):(x, y) \in P, t \in[0, \infty)\}$. The fan $\Sigma$ determines a triangulation 
of $P$ : the 1-dimensional, 2-dimensional, and 3-dimensional cones in $\Sigma$ are in oneto-one correspondence with the vertices, edges, and faces of the triangulation of $P$, respectively. This triangulation of $P$ is known as the toric diagram or the dual graph of the simplicial toric Calabi-Yau 3-fold $X_{\Sigma}$.

Let $\Sigma(d)$ be the set of $d$-dimensional cones in $\Sigma$, and let $p=|\Sigma(1)|-3$. Then $X_{\Sigma}$ is a GIT quotient

$$
X_{\Sigma}=\mathbb{C}^{3+p} / / G_{\Sigma}=\left(\mathbb{C}^{3+p}-Z_{\Sigma}\right) / G_{\Sigma}
$$

where $G_{\Sigma}$ is a $p$-dimensional subgroup of $\left(\mathbb{C}^{*}\right)^{3+p}$ and $Z_{\Sigma}$ is a Zariski closed subset of $\mathbb{C}^{3+p}$ determined by the fan $\Sigma$. If $X_{\Sigma}$ is a smooth toric Calabi-Yau 3-fold then $G_{\Sigma} \cong\left(\mathbb{C}^{*}\right)^{p}$ and $G_{\Sigma}$ acts freely on $\mathbb{C}^{3+p}-Z_{\Sigma}$. In general we have $\left(G_{\Sigma}\right)_{0} \cong\left(\mathbb{C}^{*}\right)^{p}$, where $\left(G_{\Sigma}\right)_{0}$ is the connected component of the identity, and the stabilizers of the $G_{\Sigma}$-action on $\mathbb{C}^{3+p}-Z_{\Sigma}$ are at most finite and generically trivial. The stacky quotient

$$
\mathcal{X}=\left[\left(\mathbb{C}^{3+p}-Z_{\Sigma}\right) / G_{\Sigma}\right]
$$

is a toric Calabi-Yau 3-orbifold; it is a toric Deligne-Mumford stack in the sense of Borisov-Chen-Smith [18].

2.2. Toric crepant resolution and extended Kähler classes. Given a semiprojective simplicial toric Calabi-Yau 3-fold $X_{\Sigma}$ which is not smooth, there exists a subdivision $\Sigma^{\prime}$ of $\Sigma$, such that

$$
X_{\Sigma^{\prime}}=\left(\mathbb{C}^{3+p+s}-Z_{\Sigma^{\prime}}\right) / G_{\Sigma^{\prime}} \longrightarrow X_{\Sigma}=\left(\left(\mathbb{C}^{3+p}-Z_{\Sigma}\right) \times\left(\mathbb{C}^{*}\right)^{s}\right) / G_{\Sigma^{\prime}}
$$

is a crepant toric resolution, where $X_{\Sigma^{\prime}}$ is a smooth toric Calabi-Yau 3-fold, $s=$ $\left|\Sigma^{\prime}(1)\right|-|\Sigma(1)|$, and $G_{\Sigma^{\prime}} \cong\left(\mathbb{C}^{*}\right)^{p+s}$. $X_{\Sigma^{\prime}}$ and $X_{\Sigma}$ are GIT quotients of the same $G_{\Sigma^{\prime}-\text { action on }} \mathbb{C}^{3+p+s}$ with respect to different stability conditions.

Let $K_{\Sigma^{\prime}} \cong U(1)^{p+s}$ be the maximal compact subgroup of $G_{\Sigma^{\prime}} \cong\left(\mathbb{C}^{*}\right)^{p+s}$. The $G_{\Sigma^{\prime} \text {-action on }} \mathbb{C}^{3+p+s}$ restricts to a Hamiltonian $K_{\Sigma^{\prime}}$-action on the Kähler manifold $\left(\mathbb{C}^{3+p+s}, \omega_{0}=\sqrt{-1} \sum_{i=1}^{3+p+s} d z_{i} \wedge d \bar{z}_{i}\right)$, with moment map $\widetilde{\mu}: \mathbb{C}^{3+p+s} \rightarrow \mathbb{R}^{p+s}$. There exist two (open) cones $C$ and $C^{\prime}$ in $\mathbb{R}^{p+s}$ such that

$$
\begin{aligned}
& \tilde{\mu}^{-1}(\vec{r}) / K_{\Sigma^{\prime}} \\
= & \begin{cases}\left(\mathbb{C}^{3+p+s}-Z_{\Sigma^{\prime}}\right) / G_{\Sigma^{\prime}}=X_{\Sigma^{\prime}}, & \vec{r} \in C^{\prime}, \\
\left(\left(\mathbb{C}^{3+p}-Z_{\Sigma}\right) \times\left(\mathbb{C}^{*}\right)^{s}\right) / G_{\Sigma^{\prime}}=\left(\mathbb{C}^{3+p}-Z_{\Sigma}\right) / G_{\Sigma}=X_{\Sigma}, & \vec{r} \in C\end{cases}
\end{aligned}
$$

$C^{\prime} \subset \mathbb{R}^{p+s}=H^{2}\left(X_{\Sigma^{\prime}} ; \mathbb{R}\right)$ is the Kähler cone of $X_{\Sigma^{\prime}}$ and $C \subset \mathbb{R}^{p+s}$ is the extended Kähler cone of $X_{\Sigma}$.

The parameter $\vec{r} \in C$ determines a Kähler form $\omega(\vec{r})$ on the toric Calabi-Yau 3 -orbifold $\mathcal{X}=\left[\left(\mathbb{C}^{3+p}-Z_{\Sigma}\right) / G_{\Sigma}\right]$. The $p+s$ parameters $\vec{r}=\left(r_{1}, \ldots, r_{p+s}\right)$ are extended Kähler parameters of $\mathcal{X}$, where $r_{1}, \ldots, r_{p}$ are Kähler parameters of $\mathcal{X}$. The A-model closed string flat coordinates are complexified extended Kähler parameters

$$
\tau_{a}=-r_{a}+\sqrt{-1} \theta_{a}, \quad a=1, \ldots, p+s .
$$

2.3. Toric graphs. The action of the Calabi-Yau torus $\mathbb{T}^{\prime}$ on $\mathcal{X}$ restricts to a

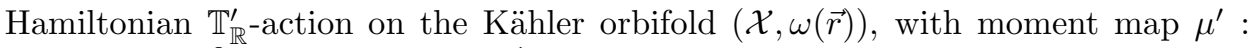
$\mathcal{X} \rightarrow M_{\mathbb{R}}^{\prime}=\mathbb{R}^{2}$. The 1 -skeleton $\mathcal{X}^{1}$ of the toric Calabi-Yau 3-fold $\mathcal{X}$ is the union of 0 -dimensional and 1-dimensional orbits of the $\mathbb{T}$-action on $\mathcal{X} . \Gamma:=\mu^{\prime}\left(\mathcal{X}^{1}\right) \subset \mathbb{R}^{2}$ is a planar trivalent graph, which is known as the toric graph of the symplectic toric Calabi-Yau 3-orbifold $(\mathcal{X}, \omega(\vec{r}))$. The toric diagram depends only on the complex 
structure on $\mathcal{X}$, where as the toric graph depends also on the symplectic structure of $\mathcal{X}$.

2.4. Aganagic-Vafa Lagrangian branes. An Aganagic-Vafa Lagrangian brane in a toric Calabi-Yau 3-orbifold $\mathcal{X}$ is a Lagrangian sub-orbifold of the form

$$
\mathcal{L}=\left[\widetilde{L} / K_{\Sigma^{\prime}}\right] \subset \mathcal{X}=\left[\widetilde{\mu}^{-1}(\vec{r}) / K_{\Sigma^{\prime}}\right]
$$

where

$$
\begin{aligned}
\widetilde{L}=\{ & \left(z_{1}, \ldots, z_{3+p+s}\right) \in \widetilde{\mu}^{-1}(\vec{r}): \\
& \left.\sum_{i=1}^{3+p+s} \hat{l}_{i}^{1}\left|z_{i}\right|^{2}=c_{1}, \sum_{i=1}^{3+p+s} \hat{l}_{i}^{2}\left|z_{i}\right|^{2}=c_{2}, \arg \left(z_{1} \cdots z_{3+p+s}\right)=c_{3}\right\},
\end{aligned}
$$

$c_{1}, c_{2}, c_{3}$ are constants, and

$$
\sum_{i=1}^{3+p+s} \hat{l}_{i}^{\alpha}=0, \quad \alpha=1,2 .
$$

The compact 2-torus $\mathbb{T}_{\mathbb{R}}^{\prime} \cong U(1)^{2}$ acts on $\mathcal{L}$, and $\mu^{\prime}(\mathcal{L})$ is a point on the toric graph $\Gamma=\mu^{\prime}\left(\mathcal{X}^{1}\right)$ which is not a vertex. $\mathcal{L}$ intersects a unique 1 -dimensional $\mathbb{T}$ orbit $\mathfrak{l} \subset \mathcal{X}$. We have $\mathfrak{l} \cong \mathbb{C}^{*} \times \mathcal{B} \mathbb{Z}_{m}$ for some positive integer $m$. When $m=1$, $\mathcal{L} \cong S^{1} \times \mathbb{C}$ is smooth; when $m>1, \mathcal{L}$ is smooth away from $\mathcal{L} \cap \mathfrak{l} \cong S^{1} \times \mathcal{B Z}_{m}$.

2.5. Chen-Ruan orbifold cohomology. Let $U=\mathbb{C}^{3+p}-Z_{\Sigma}$, so that $\mathcal{X}=$ $\left[U / G_{\Sigma}\right]$. Given $v \in G_{\Sigma}$, let $U^{v}=\{z \in U: v \cdot z=z\}$. The inertia stack of $\mathcal{X}$ is

$$
\mathcal{I X}=\bigcup_{v \in \operatorname{Box}(\Sigma)} \mathcal{X}_{v}
$$

where $\operatorname{Box}(\Sigma)=\left\{v \in G_{\Sigma}: U^{v} \neq \emptyset\right\}$ and $\mathcal{X}_{v}=\left[U^{v} / G_{\Sigma}\right]$.

We consider cohomology with $\mathbb{C}$-coefficient. As a graded $\mathbb{C}$-vector space, the Chen-Ruan orbifold cohomology [31] of $\mathcal{X}$ is

$$
H_{\mathrm{CR}}^{*}(\mathcal{X} ; \mathbb{C})=\bigoplus_{v \in \operatorname{Box}(\Sigma)} H^{*}\left(\mathcal{X}_{v} ; \mathbb{C}\right)[2 \operatorname{age}(v)], \quad \operatorname{age}(v) \in\{0,1,2\}
$$

Let $\mathfrak{g}:=\left|\operatorname{Int}(P) \cap N^{\prime}\right|$ be the number of lattice points in $\operatorname{Int}(P)$, the interior of the polytope $P$, and let $\mathfrak{n}:=\left|\partial P \cap N^{\prime}\right|$ be the number of lattice points on $\partial P$, the boundary of the polytope $P$. Then

$$
\begin{aligned}
p & =|\Sigma(1)|-3=\operatorname{dim}_{\mathbb{C}} H^{2}\left(X_{\Sigma} ; \mathbb{C}\right), \\
p+s & =\left|\Sigma^{\prime}(1)\right|-3=\left|P \cap N^{\prime}\right|-3=\operatorname{dim}_{\mathbb{C}} H^{2}\left(X_{\Sigma^{\prime}} ; \mathbb{C}\right)=\operatorname{dim}_{\mathbb{C}} H_{\mathrm{CR}}^{2}(\mathcal{X} ; \mathbb{C}) \\
& =\mathfrak{g}+\mathfrak{n}-3, \\
\mathfrak{g} & =\left|\operatorname{Int}(P) \cap N^{\prime}\right|=\operatorname{dim}_{\mathbb{C}} H^{4}\left(X_{\Sigma^{\prime}}\right)=\operatorname{dim}_{\mathbb{C}} H_{\mathrm{CR}}^{4}(\mathcal{X} ; \mathbb{C}), \\
\chi & =\left|\Sigma^{\prime}(3)\right|=2 \operatorname{Area}(P)=\operatorname{dim}_{\mathbb{C}} H^{*}\left(X_{\Sigma^{\prime}} ; \mathbb{C}\right)=\operatorname{dim}_{\mathbb{C}} H_{\mathrm{CR}}^{*}(\mathcal{X} ; \mathbb{C}) \\
& =1+p+s+\mathfrak{g}=2 \mathfrak{g}-2+\mathfrak{n} .
\end{aligned}
$$


2.6. The mirror curve. Following the notation in Section 2.5, the convex polytope $P \subset N_{\mathbb{R}}^{\prime} \cong \mathbb{R}^{2}$ defines a polarized toric surface $(S, L)$, where $S$ is a toric variety and $L$ is an ample line bundle. We have

$$
\chi(S, L)=h^{0}(S, L)=\left|P \cap N^{\prime}\right|=3+p+s .
$$

The mirror curve $H(X, Y)$ is given by

$$
H(X, Y)=\sum_{(m, n) \in P \cap N^{\prime}} a_{m, n} X^{m} Y^{n}, a_{m, n} \in \mathbb{C}^{*} .
$$

So $H(X, Y) \in H^{0}\left(\left(\mathbb{C}^{*}\right)^{2}, \mathcal{O}_{\left(\mathbb{C}^{*}\right)^{2}}\right)$ is the restriction of a section $s \in H^{0}(S, L)$. The compactified mirror curve is $s^{-1}(0) \subset S$.

The element $\left(t_{1}, t_{2}, t_{3}\right) \in\left(\mathbb{C}^{*}\right)^{3}$ acts on the section $H(X, Y)$ by

$$
H(X, Y) \mapsto t_{3} H\left(t_{1} X, t_{2} Y\right)
$$

Modulo this action, the mirror curve is parametrized by $p+s$ elements $q=$ $\left(q_{1}, \ldots, q_{p+s}\right) \in\left(\mathbb{C}^{*}\right)^{p+s}$. For generic $q$, the mirror curve $C_{q}$ is a Riemann surface of genus $\mathfrak{g}$ with $\mathfrak{n}$ punctures, and the compactified mirror curve $\bar{C}_{q}$ is a smooth hypersurface in the toric surface $S$. The Euler characteristic of $C_{q}$ is

$$
\chi\left(C_{q}\right)=2-2 \mathfrak{g}-\mathfrak{n}=-\operatorname{dim}_{\mathbb{C}} H^{*}(\mathcal{X} ; \mathbb{C})=-\chi(X) .
$$

2.7. Framings. The framing $f \in \mathbb{Z}$ specifies a 1 -dimensional subgroup

$$
\mathbb{T}_{f}=\operatorname{ker}(\mathrm{f}) \subset \mathbb{T}^{\prime},
$$

where the character $\mathrm{f}=\mathrm{w}_{1}^{\prime}-f \mathrm{w}_{2}^{\prime} \in M^{\prime}=\operatorname{Hom}\left(N^{\prime}, \mathbb{Z}\right)$. It induces a surjective group homomorphism

$$
\left(\mathbb{T}^{\prime}\right)^{\vee} \cong\left(\mathbb{C}^{*}\right)^{2} \rightarrow\left(\mathbb{T}_{f}\right)^{\vee} \cong \mathbb{C}^{*}, \quad(X, Y) \mapsto X Y^{f} .
$$

Other than several finite number of choices of $f$, the function

$$
\hat{X}:=X Y^{f}: C_{q} \rightarrow \mathbb{C}^{*}
$$

is holomorphic Morse, i.e. it has simple ramification points. We have

$$
|\operatorname{Crit}(\hat{X})|=-\chi\left(C_{q}\right)=2 \mathfrak{g}-2+\mathfrak{n}=\operatorname{dim}_{\mathbb{C}} H_{\mathrm{CR}}^{*}(\mathcal{X} ; \mathbb{C}) .
$$

Around each ramification point $p_{0} \in \operatorname{Crit}(\hat{X})$, one writes

$$
\hat{x}=\hat{x}\left(p_{0}\right)+\zeta_{0}^{2}
$$

where $\zeta_{0}$ is the local coordinate around $p_{0}$. We denote $\check{u}^{p_{0}}=\hat{x}\left(p_{0}\right)$. It depends on the complex parameter $q$, and is a canonical coordinate of the B-model. For any $p$ in the neighborhood of $p_{0}$ we define $\bar{p}$ by

$$
\zeta_{0}(p)=-\zeta_{0}(\bar{p}) .
$$

We also define a multi-valued holomorphic 1-form on $C_{q}$

$$
\Phi=\log Y \frac{d \hat{X}}{\hat{X}} .
$$




\section{Gromov-Witten invariants of Toric Calabi-Yau 3-orbifolds}

3.1. Open Gromov-Witten invariants and A-model open potentials. Let $\mathcal{L}$ be an Aganagic-Vafa Lagrangian brane in a toric Calabi-Yau 3-orbifold $\mathcal{X}$. Then $\mathcal{L}$ is homotopic to $S^{1} \times \mathcal{B} \mathbb{Z}_{m}$, so

$$
H_{1}(\mathcal{L} ; \mathbb{Z})=\pi_{1}(\mathcal{L})=\mathbb{Z} \times \mathbb{Z}_{m}
$$

Open $\mathrm{GW}$ invariants of $(\mathcal{X}, \mathcal{L})$ count holomorphic maps

$$
u:\left(\Sigma, x_{1}, \ldots, x_{\ell}, \partial \Sigma=\coprod_{j=1}^{n} R_{j}\right) \rightarrow(\mathcal{X}, \mathcal{L})
$$

where $\Sigma$ is a bordered Riemann surface with stacky points $x_{i}=B \mathbb{Z}_{r_{i}}$ and $R_{j} \cong S^{1}$ are connected components of $\partial \Sigma$. These invariants depend on the following data:

(1) the topological type $(g, n)$ of the coarse moduli of the domain, where $g$ is the genus of $\Sigma$ and $n$ is the number of connected components of $\partial \Sigma$,

(2) the degree $\beta^{\prime}=u_{*}[\Sigma] \in H_{2}(\mathcal{X}, \mathcal{L} ; \mathbb{Z})$,

(3) the winding numbers $\mu_{1}, \ldots, \mu_{n} \in \mathbb{Z}$ and the monodromies $k_{1}, \ldots, k_{n} \in \mathbb{Z}_{m}$, where $\left(\mu_{j}, k_{j}\right)=u_{*}\left[R_{j}\right] \in H_{1}(\mathcal{L} ; \mathbb{Z})=\mathbb{Z} \times \mathbb{Z}_{m}$,

(4) the framing $f \in \mathbb{Z}$ of $\mathcal{L}$.

We call the pair $(\mathcal{L}, f)$ a framed Aganagic-Vafa Lagrangian brane. We write $\vec{\mu}=$ $\left(\left(\mu_{1}, k_{1}\right), \ldots,\left(\mu_{n}, k_{n}\right)\right)$. Let $\mathcal{M}_{g, \ell}\left(\mathcal{X}, \mathcal{L} \quad \mid \beta^{\prime}, \vec{\mu}\right)$ be the moduli space parametrizing maps described above, and let $\overline{\mathcal{M}}_{g, \ell}\left(\mathcal{X}, \mathcal{L} \mid \beta^{\prime}, \vec{\mu}\right)$ be the partial compactification: we allow the domain $\Sigma$ to have nodal singularities, and an orbifold/stacky point on $\Sigma$ is either a marked point $x_{j}$ or a node; we require the map $u$ to be stable in the sense that its automorphism group is finite. Evaluation at the $i$-th marked point $x_{i}$ gives a map ev $i: \overline{\mathcal{M}}_{(g, n), \ell}\left(\mathcal{X}, \mathcal{L} \mid \beta^{\prime}, \vec{\mu}\right) \rightarrow \mathcal{I} \mathcal{X}$.

Given $\gamma_{1}, \ldots, \gamma_{\ell} \in H_{\mathrm{CR}, \mathbb{T}^{\prime}}^{*}(\mathcal{X} ; \mathbb{C})$, we define

$$
\begin{aligned}
\left\langle\gamma_{1}, \ldots, \gamma_{\ell}\right\rangle_{g, \beta, \vec{\mu}}^{\mathcal{X},(\mathcal{L}, f)} & :=\left.\int_{\left[\overline{\mathcal{M}}_{(g, n), \ell}\left(\mathcal{X}, \mathcal{L} \mid \beta^{\prime}, \vec{\mu}\right)^{\mathbb{T}_{\mathbb{R}}^{\prime}}\right]^{\mathrm{vir}}} \frac{\prod_{i=1}^{\ell} \mathrm{ev}_{i}^{*} \gamma_{i}}{e_{\mathbb{T}_{\mathbb{R}}^{\prime}}\left(N^{\mathrm{vir}}\right)}\right|_{\left(\mathbb{T}_{f}\right)_{\mathbb{R}}} \\
& \in \mathbb{C} v^{\sum_{i=1}^{\ell} \frac{\operatorname{deg} \gamma_{i}}{2}-1}
\end{aligned}
$$

where $v$ is the generator of $H^{2}\left(\mathcal{B}\left(\mathbb{T}_{f}\right)_{\mathbb{R}} ; \mathbb{Z}\right)=H^{2}(\mathcal{B U}(1) ; \mathbb{Z}) \cong \mathbb{Z}$.

For $\boldsymbol{\tau}=\sum_{a=1}^{p+s} \tau_{a} e_{a} \in H_{\mathrm{CR}}^{2}(\mathcal{X} ; \mathbb{C})$, we define generating functions $F_{g, n}^{\mathcal{X},(\mathcal{L}, f)}$ of open Gromov-Witten invariants as follows.

$$
\begin{aligned}
& F_{g, n}^{\mathcal{X},(\mathcal{L}, f)}\left(Z_{1}, \ldots, Z_{n}, \boldsymbol{\tau}\right) \\
&= \sum_{\beta^{\prime}, \ell \geq 0} \sum_{\left(\mu_{j}, k_{j}\right) \in \mathbb{Z} \times \mathbb{Z}_{m}} \frac{\left\langle\boldsymbol{\tau}^{\ell}\right\rangle_{g, \beta,\left(\mu_{1}, k_{1}\right) \cdots,\left(\mu_{n}, k_{n}\right)}^{\mathcal{X},(\mathcal{L}, f)}}{\ell !} \\
& \cdot \otimes_{j=1}^{n}\left(Z_{j}^{\mu_{j}}\left(-(-1)^{\frac{-k_{j}}{m}}\right) \mathbf{1}_{\frac{{ }_{-k_{j}}}{m}}^{\prime}\right) \in H_{\mathrm{CR}}^{*}\left(\mathcal{B} \mathbb{Z}_{m} ; \mathbb{C}\right)^{\otimes n}
\end{aligned}
$$

where $H_{\mathrm{CR}}^{*}\left(\mathcal{B} \mathbb{Z}_{m} ; \mathbb{C}\right)=\oplus_{k=0}^{m-1} \mathbb{C} \mathbf{1}_{\frac{k}{m}}^{\prime}$. 


\subsection{Primary closed Gromov-Witten invariants and}

A-model free energies. We define genus $g$, degree $\beta$ primary closed GromovWitten invariants:

$$
\left\langle\boldsymbol{\tau}^{\ell}\right\rangle_{g, \beta}^{\mathcal{X}}=\left.\int_{\overline{\mathcal{M}}_{g, \ell}(\mathcal{X}, \beta)^{\left.\mathbb{T}_{\mathbb{R}}^{\prime}\right]^{\mathrm{vir}}}} \frac{\prod_{i=1}^{\ell} \mathrm{ev}_{i}^{*} \boldsymbol{\tau}}{e_{\mathbb{T}_{\mathbb{R}}^{\prime}}\left(N^{\text {vir }}\right)}\right|_{\left(\mathbb{T}_{f}\right)_{\mathbb{R}}} \in \mathbb{C} .
$$

This closed Gromov-Witten invariant can be viewed as the case when $n=0$ i.e. there is no boundary on the domain curve. The A-model genus $g$ free energy $F_{g}^{\mathcal{X}}$ is a generating function of primary genus $g$ closed Gromov-Witten invariants.

$$
F_{g}^{\mathcal{X}}(\boldsymbol{\tau})=\sum_{\beta, \ell \geq 0} \frac{\left\langle\boldsymbol{\tau}^{\ell}\right\rangle_{g, \beta}^{\mathcal{X}}}{\ell !}
$$

The BKMP remodeling conjecture builds the mirror symmetry for the open Gromov-Witten potentials $F_{g, n}^{\mathcal{X},(\mathcal{L}, f)}\left(Z_{1}, \ldots, Z_{n}, \boldsymbol{\tau}\right)$ as well as free energies $F_{g}^{\mathcal{X}}(\boldsymbol{\tau})$.

3.3. Descendant closed Gromov-Witten invariants. Given $\gamma_{1}, \ldots, \gamma_{n}$, we define a generating function of genus $g, n$-point descendant closed Gromov-Witten invariants:

$$
\left\langle\left\langle\frac{\gamma_{1}}{z_{1}-\psi_{1}}, \ldots, \frac{\gamma_{n}}{z_{n}-\psi_{n}}\right\rangle_{g, n}^{\mathcal{X}}=\sum_{\beta, \ell \geq 0} \frac{1}{\ell !}\left\langle\frac{\gamma_{1}}{z_{1}-\psi_{1}}, \ldots, \frac{\gamma_{n}}{z_{n}-\psi_{n}}, \boldsymbol{\tau}^{\ell}\right\rangle_{g, \beta}^{\mathcal{X}},\right.
$$

where $\psi_{i}=c_{1}\left(\mathbb{L}_{i}\right)$ and $\mathbb{L}_{i} \rightarrow \overline{\mathcal{M}}_{g, n+\ell}(\mathcal{X}, \beta)$ is line bundle whose fiber at moduli point $\left[u:\left(C, x_{1}, \ldots, x_{n+\ell}\right) \rightarrow \mathcal{X}\right]$ is the cotangent line $T_{x_{i}}^{*} C$ at the $i$-th marked point to (the coarse moduli space of) the domain curve.

We will state an extension of the remodeling conjecture to higher genus descendent potentials $\left\langle\frac{\gamma_{1}}{z_{1}-\psi_{1}}, \ldots, \frac{\gamma_{n}}{z_{n}-\psi_{n}}\right\rangle_{g, n}^{\mathcal{X}}$.

\section{Eynard-Orantin invariants of the Mirror CURVE}

4.1. Fundamental normalized differential of the second kind. In this subsection, we recall the definition of the fundamental normalized differential of the second kind $B\left(p_{1}, p_{2}\right)$ (see e.g. [53]) for a general compact Riemann surface $\bar{C}$.

Let $\bar{C}$ be a compact Riemann surface of genus $\mathfrak{g}$. When $\mathfrak{g}>0$, let $A_{1}, B_{1}$, $\ldots, A_{\mathfrak{g}}, B_{\mathfrak{g}}$ be a symplectic basis of $\left(H_{1}(\bar{C} ; \mathbb{C}), \cdot\right)$ :

$$
A_{i} \cdot A_{j}=B_{i} \cdot B_{j}=0, \quad A_{i} \cdot B_{j}=-B_{j} \cdot A_{i}=\delta_{i j}
$$

where $\cdot$ is the intersection pairing. For our purpose, we need to consider $H_{1}(\bar{C} ; \mathbb{C})$ instead of the integral first homology group $H_{1}(\bar{C} ; \mathbb{Z})$. We assume that the Lagrangian subspace $\bigoplus_{i=1}^{\mathfrak{g}} \mathbb{C} A_{i}$ of $H_{1}(\bar{C} ; \mathbb{C})$ is transversal to the Lagrangian subspace

$$
H^{1,0}(\bar{C})^{\perp}:=\left\{\gamma \in H_{1}(\bar{C} ; \mathbb{C}):\langle\theta, \gamma\rangle=0 \quad \forall \theta \in H^{1,0}(\bar{C})\right\}
$$

where $\langle\rangle:, H^{1}(\bar{C} ; \mathbb{C}) \times H_{1}(\bar{C} ; \mathbb{C})$ is the natural pairing; this assumption holds when $A_{1}, \ldots, A_{\mathfrak{g}} \in H_{1}(\bar{C} ; \mathbb{R})$.

The fundamental normalized differential of the second kind $B\left(p_{1}, p_{2}\right)$ on $\bar{C}$ is characterized by the following properties:

(1) $B\left(p_{1}, p_{2}\right)$ is a bilinear symmetric meromorphic differential on $\bar{C}_{q} \times \bar{C}_{q}$. 
(2) $B\left(p_{1}, p_{2}\right)$ is holomorphic everywhere except for a double pole along the diagonal. If $p_{1}, p_{2}$ have local coordinates $z_{1}, z_{2}$ in an open neighborhood $U$ of $p \in \bar{C}_{q}$ then

$$
B\left(p_{1}, p_{2}\right)=\left(\frac{1}{\left(z_{1}-z_{2}\right)^{2}}+a\left(z_{1}, z_{2}\right)\right) d z_{1} d z_{2}
$$

where $a\left(z_{1}, z_{2}\right)$ is holomorphic on $U \times U$ and symmetric in $z_{1}, z_{2}$.

(3) $\int_{p_{1} \in A_{i}} B\left(p_{1}, p_{2}\right)=0, i=1, \ldots, \mathfrak{g}$.

In fact, we do not need a particular choice of A-cycles. We just need to specify a Lagrangian subspace of $\left(H_{1}(\bar{C} ; \mathbb{C}), \cdot\right)$ transversal to the Lagrangian subspace $H^{1,0}(\bar{C} ; \mathbb{C})^{\perp} \subset H_{1}(\bar{C} ; \mathbb{C})$ such that the period of $B\left(p_{1}, p_{2}\right)$ along any element in this subspace is zero.

The fundamental differential $B\left(p_{1}, p_{2}\right)$ also satisfies the following properties:

(4) If $f$ is a meromorphic function on $\bar{C}$ then

$$
d f\left(p_{1}\right)=\operatorname{Res}_{p_{2} \rightarrow p_{1}} B\left(p_{1}, p_{2}\right) f\left(p_{2}\right) .
$$

(5) $\int_{p_{1} \in B_{i}} B\left(p_{1}, p_{2}\right)=2 \pi \sqrt{-1} \omega_{i}\left(p_{2}\right)$, where $\omega_{i}$ is the unique holomorphic 1form on $\bar{C}$ such that $\int_{A_{j}} \omega_{i}=\delta_{i j}$.

4.2. Choice of A-cycles on the compactified mirror curve. The mirror theorem for semi-projective toric orbifolds 33 relates the 1-primary 1-descendant function (the $J$-function)

$$
\|\left\langle 1, \frac{\phi_{a}}{z-\psi} \|_{0,2}^{\mathcal{X}} \phi^{a}\right.
$$

to certain hypergeometric $I$-function $I^{\mathcal{X}}(q, z)$ under the mirror map

$$
\tau_{a}=\frac{1}{2 \pi \sqrt{-1}} \int_{A_{a}} \Phi= \begin{cases}\log q_{a}+h_{a}(q), & a=1, \ldots, p \\ q_{a}\left(1+h_{a}(q)\right), & a=p+1, \ldots, p+s .\end{cases}
$$

which as the prescribed leading term behavior (all $h_{a}(q)$ are power series in $\left.q\right)$.

It is a well-known fact that these mirror maps are given by such period integrals where $A_{a} \in H_{1}\left(C_{q} ; \mathbb{C}\right)$. The inclusion $C_{q} \hookrightarrow \bar{C}_{q}$ induces a surjective group homomorphism $H_{1}\left(C_{q} ; \mathbb{C}\right) \cong \mathbb{C}^{2 \mathfrak{g}+\mathfrak{n}-1} \rightarrow H_{1}\left(\bar{C}_{q}\right) \cong \mathbb{C}^{2 \mathfrak{g}}$ where the kernel is generated by the $\mathfrak{n}$ loops around the $\mathfrak{n}$ points in $\bar{C}_{q} \backslash C_{q}$; each of these $\mathfrak{n}$ loops is contractible in $\bar{C}_{q}$, and the sum of these $\mathfrak{n}$ loops is homologous to zero in $C_{q}$. The images of $A_{a} \in H_{1}\left(C_{q} ; \mathbb{C}\right)$ in $H_{1}\left(\bar{C}_{q} ; \mathbb{C}\right)$ span a Lagrangian subspace $L_{A} \subset H_{1}\left(\bar{C}_{q} ; \mathbb{C}\right)$ transversal to the Lagrangian subspace $H^{1,0}\left(\bar{C}_{q}\right)^{\perp} \subset H_{1}\left(\bar{C}_{q} ; \mathbb{C}\right)$. We use the Lagrangian subspace $L_{A}$ to define our fundamental normalized differential of the second kind $B\left(p_{1}, p_{2}\right)$ for the purpose of constructing higher genus B-model invariants.

4.3. The Eynard-Orantin topological recursion. We use the fundamental differential $B$ prescribed above to run the Eynard-Orantin topological recursion. It starts with two initial data (unstable cases)

$$
\omega_{0,1}=0, \quad \omega_{0,2}=B .
$$


The stable cases $(2 g-2+n>0)$ are defined recursively by the Eynard-Orantin topological recursion:

$$
\begin{aligned}
& \omega_{g, n}\left(p_{1}, \ldots, p_{n}\right) \\
:= & \sum_{p_{0} \in \operatorname{Crit}(\hat{X})} \operatorname{Res}_{p \rightarrow p_{0}} \frac{\int_{\xi=p}^{\bar{p}} B\left(p_{n}, \xi\right)}{2(\Phi(p)-\Phi(\bar{p}))}\left(\omega_{g-1, n+1}\left(p, \bar{p}, p_{1}, \ldots, p_{n-1}\right)\right. \\
& \left.+\sum_{\substack{g_{1}+g_{2}=g \\
I \sqcup J=\{1, \ldots, n-1\}}} \omega_{g_{1},|I|+1}\left(p, p_{I}\right) \omega_{g_{2},|J|+1}\left(\bar{p}, p_{J}\right)\right) .
\end{aligned}
$$

The resulting $\omega_{g, n}$ for $2 g-2+n>0$ is a symmetric meromorphic form on $\left(\bar{C}_{q}\right)^{n}$. They are holomorphic on $\left(\bar{C}_{q} \backslash \operatorname{Crit}(\hat{X})\right)^{n}$ and satisfy the following properties:

(1) For any $j \in\{1, \ldots, n\}$ and any $p_{0} \in \operatorname{Crit}(\hat{X})$,

$$
\operatorname{Res}_{p_{j} \rightarrow p_{0}} \omega_{g, n}\left(p_{1}, \ldots, p_{n}\right)=0 .
$$

(2) For any $j \in\{1, \ldots, n\}$ and any $i \in\{1, \ldots, \mathfrak{g}\}$,

$$
\int_{p_{j} \in A_{i}} \omega_{g, n}\left(p_{1}, \ldots, p_{n}\right)=0
$$

4.4. B-model open potentials. For $\ell \in \mathbb{Z}_{m}=\mathbb{Z} / m \mathbb{Z}$, let

$$
\psi_{\ell}:=\frac{1}{m} \sum_{k=0}^{m-1} e^{\frac{2 \pi \sqrt{-1} k \ell}{m}} \mathbf{1}_{\frac{k}{m}}^{\prime} .
$$

Then $\left\{\psi_{\ell}: \ell=0,1 \ldots, m-1\right\}$ is a canonical basis of $H_{\mathrm{CR}}^{*}\left(\mathcal{B Z}_{m} ; \mathbb{C}\right)$.

Recall that $\mathcal{L}$ intersects a unique 1 -dimensional orbit $\mathfrak{l}$ of the $\mathbb{T}$-action on $\mathcal{X}$. We assume that the closure $\overline{\mathfrak{l}}$ of $\mathfrak{l}$ in $\mathcal{X}$ is non-compact, so that $\mathcal{L}$ is an "outer" brane. Then the 2-dimensional cone associated to $\overline{\mathfrak{l}}$ corresponds an edge $e$ on the boundary of the polytope $P$, and $\left|e \cap N^{\prime}\right|=m+1$. Let $D \subset S$ be the torus invariant divisor associated to the edge $e$. For generic $q$, the compactified mirror curve $\bar{C}_{q}$ intersects $D$ transversally at $m$ points $\bar{p}_{0}, \ldots, \bar{p}_{m-1}$. For $\ell \in\{0,1, \ldots, m-1\}$, there exist open neighborhoods $U_{\ell}$ of $\bar{p}_{\ell}$ in the compactified mirror curve $\bar{C}_{q}$ and $U$ of 0 in $\mathbb{P}^{1}=\mathbb{C}^{*} \cup\{0, \infty\}$ such that $\left.\hat{X}\right|_{U_{\ell}}: U_{\ell} \rightarrow U$ is biholomorphic. Let $\rho_{\ell}:=\left(\left.\hat{X}\right|_{U_{\ell}}\right)^{-1}: U \rightarrow U_{\ell}$. We define B-model topological open string partition functions as follows.

(1) disk invariants

$$
\check{F}_{0,1}(q ; X):=\sum_{\ell \in \mathbb{Z}_{m}} \int_{0}^{X}\left(\left(\log Y\left(\rho_{\ell}\left(X^{\prime}\right)\right)-\log Y\left(\bar{p}_{\ell}\right)\right) \frac{d X^{\prime}}{X^{\prime}}\right) \psi_{\ell}
$$

which take values in $H_{\mathrm{CR}}^{*}\left(\mathcal{B} \mathbb{Z}_{m} ; \mathbb{C}\right)$.

(2) annulus invariants

$$
\begin{aligned}
& \check{F}_{0,2}\left(q ; X_{1}, X_{2}\right) \\
:= & \sum_{\ell_{1}, \ell_{2} \in \mathbb{Z}_{m}} \int_{0}^{X_{1}} \int_{0}^{X_{2}}\left(\left(\rho_{\ell_{1}} \times \rho_{\ell_{2}}\right)^{*} \omega_{0,2}-\frac{d X_{1}^{\prime} d X_{2}^{\prime}}{\left(X_{1}^{\prime}-X_{2}^{\prime}\right)^{2}}\right) \psi_{\ell_{1}} \otimes \psi_{\ell_{2}}
\end{aligned}
$$

which take values in $H_{\mathrm{CR}}^{*}\left(\mathcal{B} \mathbb{Z}_{m} ; \mathbb{C}\right)^{\otimes 2}$. 
(3) $2 g-2+n>0$

$$
\begin{aligned}
& \check{F}_{g, n}\left(q ; X_{1}, \ldots, X_{n}\right) \\
:= & \sum_{\ell_{1}, \cdots, \ell_{n} \in \mathbb{Z}_{m}} \int_{0}^{X_{1}} \cdots \int_{0}^{X_{n}}\left(\rho_{\ell_{1}} \times \cdots \times \rho_{\ell_{n}}\right)^{*} \omega_{g, n} \psi_{\ell_{1}} \otimes \cdots \otimes \psi_{\ell_{n}}
\end{aligned}
$$

which take values in $H_{\mathrm{CR}}^{*}\left(\mathcal{B} \mathbb{Z}_{m} ; \mathbb{C}\right)^{\otimes n}$.

Each of the $m^{n}$ components of $\check{F}_{g, n}\left(q ; X_{1}, \ldots, X_{n}\right)$ is a power series in $q_{1}, \ldots, q_{p+s}, X_{1}, \ldots, X_{n}$ which converges in an open neighborhood of the origin.

4.5. B-model free energies. For $g \geq 2$, the B-model free energy is defined as

$$
\check{F}_{g}(q)=\frac{1}{2 g-2} \sum_{p_{0} \in \operatorname{Crit} \hat{X}} \operatorname{Res}_{p \rightarrow p_{0}} \omega_{g, 1}(p) \widetilde{\Phi}(p),
$$

where

$$
d \widetilde{\Phi}=\Phi .
$$

Notice that the function $\widetilde{\Phi}$ locally defined around each critical point of $\hat{X}$ has some ambiguities, since $\Phi$ is multi-valued, and $\widetilde{\Phi}$ is determined by $\Phi$ up to a constant. However, the residue is well-defined since is does not depend on these ambuities.

For $g=1$, the free energy is defined up to a constant

$$
\check{F}_{1}(q)=-\frac{1}{2} \log \tau_{B}-\frac{1}{24} \sum_{p_{0} \in \text { Crit } \hat{X}} \log h_{1}^{p_{0}} .
$$

Here the Bergmann $\tau$-function $\tau_{B}$ is defined up to a constant by

$$
\frac{\partial \log \tau_{B}}{\partial \check{u}^{p_{0}}}=\operatorname{Res}_{p \rightarrow p_{0}} \frac{B(p, \bar{p})}{d \hat{x}(p)} .
$$

When $g=0$, the prepotential $F_{0}$ is characterized by

$$
\frac{\partial \check{F}_{0}}{\partial \tau_{a}}=\int_{p \in B_{a}} \Phi(p)
$$

Notice that since $\Phi$ is a multi-valued differential form, and it satisfies the following

$$
\frac{\partial \Phi(p)}{\partial \tau_{a}}=\int_{p^{\prime} \in B_{a}} \omega_{0,2}\left(p, p^{\prime}\right) .
$$

The prepotential $\check{F}_{0}$ defined this way is only determined up to a quadratic polynomial in $\tau_{a}$.

\section{All genus open-Closed MirRor SyMmetry}

In this section, $(\mathcal{L}, f)$ is an outer Aganagic-Vafa Lagrangian brane in $\mathcal{X}$, so that the closure of $\mathfrak{l}=\mathbb{C}^{*} \times \mathcal{B} \mathbb{Z}_{m}$ contains a unique $\mathbb{T}$ fixed point. Let $G$ be the stabilizer of this fixed point. Then $G$ is a finite abelian group which contains $\mathbb{Z}_{m}$ as a subgroup. When $\mathcal{X}$ is smooth, we have $m=1$ and $G$ is trivial. 


\subsection{All genus open-closed mirror symmetry: the remodeling conjecture.}

Conjecture 1 (Bouchard-Klemm-Mariño-Pasqetti [20, 21]).

$$
\check{F}_{g, n}\left(q ; X_{1}, \ldots, X_{n}\right)=(-1)^{g-1+n}|G|^{n} F_{g, n}^{\mathcal{X},(\mathcal{L}, f)}\left(\boldsymbol{\tau} ; Z_{1}, \ldots, Z_{n}\right)
$$

where $\left(q, X_{j}\right)$ and $\left(\boldsymbol{\tau}, Z_{j}\right)$ are related by the open-closed mirror map:

$$
\begin{aligned}
\tau_{a}=\frac{1}{2 \pi \sqrt{-1}} \int_{A_{a}} \Phi & = \begin{cases}\log q_{a}+h_{a}(q), & a=1 \ldots, p \\
q_{a}\left(1+h_{a}(q)\right), & a=p+1, \ldots, p+s\end{cases} \\
\log Z_{j} & =\log X_{j}+h_{0}(q)
\end{aligned}
$$

where $h_{0}(q), h_{1}(q), \ldots, h_{p+s}(q)$ are explicit power series in $q$ convergent in a neighborhood of the origin in $\mathbb{C}^{p+s}$. Notice that when $n=0$, this is a statement about closed Gromov-Witten mirror symmetry (and the right-hand side does not depend on $(\mathcal{L}, f))$. When $(g, n)=(1,0)$ and $(0,0)$, the statement is valid up to a constant and a quadratic polynomial in $\tau_{a}$, respectively.

Indeed, the above statement is more general than the original conjecture in 20, 21, where they conjecture about non-gerby branes (the $m=1$ case).

Conjecture 1 was proved when $\mathcal{X}=\mathbb{C}^{3}$ independently by L. Chen [28] and J. Zhou [116. In 2012, Eynard-Orantin provided a proof of the BKMP remodeling conjecture for all symplectic smooth toric Calabi-Yau 3-folds [47. In the orbifold case, the authors prove Conjecture 1 first for affine toric Calabi-Yau 3-orbifolds [51. and later for all semi-projective toric Calabi-Yau 3-orbifolds 52.

We now give a brief outline of the proof of Conjecture 1 in [52]. Givental proved a quantization formula for total descendant potential of equivariant GW theory of GKM manifolds [57, 58, 59]. (See also the book by Lee-Pandharipande [77].) The third author generalized this formula to GKM orbifolds [120. The quantization formula is equivalent to a graph sum formula of the total descendant potential, which implies a graph sum formula

$$
F_{g, n}^{\mathcal{X},(\mathcal{L}, f)}=\sum_{\vec{\Gamma} \in G_{g, n}} \frac{w_{A}(\vec{\Gamma})}{|\operatorname{Aut}(\vec{\Gamma})|},
$$

where $G_{g, n}$ is a certain set of decorated stable graphs. The unique solution $\left\{\omega_{g, n}\right\}$ to the Eynard-Orantin topological recursion can be expressed as a sum over graphs [70, 44, 45, 43. We expand the graph sum formula in [43, Theorem 3.7] (which is equivalent to [44. Theorem 5.1]) at punctures $\left\{\bar{p}_{\ell}: \ell \in \mathbb{Z}_{m}\right\}$, and obtain a graph sum formula

$$
\check{F}_{g, n}=\sum_{\vec{\Gamma} \in G_{g, n}} \frac{w_{B}(\vec{\Gamma})}{|\operatorname{Aut}(\vec{\Gamma})|} .
$$

Finally, we use the genus-zero mirror theorem for smooth toric DM stacks [33] to prove

$$
w_{B}(\vec{\Gamma})=(-1)^{g-1+n}|G|^{n} w_{A}(\vec{\Gamma})
$$

for all decorated graphs $\vec{\Gamma}$. 
5.2. Descendant version of the all genus mirror symmetry. Iritani 68] studies the oscillatory integral and shows the following

$$
\int_{\mathrm{SYZ}(\mathcal{F})} e^{\frac{\widetilde{W}}{z}} \Omega=\|\left\langle\frac{\kappa(\mathcal{F})}{z-\psi} \|_{0,1},\right.
$$

where $\mathcal{F}$ is a $\mathbb{T}_{f}$-equivariant coherent sheaf on $\mathcal{X}$. Here the SYZ is the SYZ Tdual functor, which takes a $\mathbb{T}_{f}$-equivariant coherent sheaf on $\mathcal{X}$ and produces a Lagrangian brane in $\left(\mathbb{C}^{*}\right)^{3} 11$ The equivariantly perturbed superpotential $W$ is given by

$$
\widetilde{W}=W-\log X-f \log Y .
$$

Let $\left(\mathbb{T}_{f}\right)_{\mathbb{R}} \cong U(1)$ be the maximal torus of $\mathbb{T}_{f} \cong \mathbb{C}^{*}$, and let $\mu_{\left(\mathbb{T}_{f}\right)_{\mathbb{R}}}: \mathcal{X} \rightarrow \mathbb{R}$

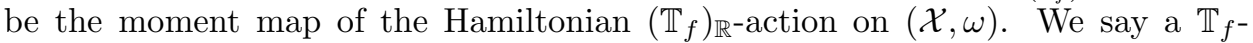
equivariant coherent sheaf $\mathcal{F}$ on $\mathcal{X}$ is admissible if (i) $\mu_{\left(\mathbb{T}_{f}\right)_{\mathbb{R}}}(\operatorname{supp}(\mathcal{F})) \subset \mathbb{R}$ is bounded below, and (ii) the Lagrangian brane $\operatorname{SYZ}(\mathcal{F})$ reduces to a cycle $\gamma(\mathcal{F})$ on the mirror curve $C_{q}$, while the oscillatory integral could be done on the curve

$$
\int_{\mathrm{SYZ}(L)} e^{\frac{\widetilde{W}}{z}} \frac{d X d Y d Z}{X Y Z}=\int_{\gamma(\mathcal{F})} e^{\frac{\hat{x}}{z}} y d x .
$$

Condition (i) implies that $\hat{x}$ is bounded below on $\gamma(\mathcal{F})$, so the integral on the RHS converges when $z \in(-\infty, 0)$.

Using this result and combining with the remodeling conjecture, we have

Theorem 2 (Descendant version of the all genus mirror symmetry for $\mathcal{X}$ ).

$$
\int_{\gamma\left(L_{1}\right) \times \cdots \times \gamma\left(L_{n}\right)} e^{\frac{\hat{x}_{1}}{z_{1}}+\cdots+\frac{\hat{x}_{n}}{z_{n}}} \omega_{g, n}=\left\langle\frac{\kappa\left(L_{1}\right)}{z_{1}-\psi_{1}} \cdots \frac{\kappa\left(L_{n}\right)}{z_{n}-\psi_{n}} \|_{g, n} .\right.
$$

To obtain this theorem, one observes that when integrating $\omega_{g, n}$ we are simply integrating the leaf terms of $w_{A}(\vec{\Gamma})$, since only leaf terms are forms while all other graph component contributions are scalars. The genus 0 oscillatory integral theorem from [68] turns these leafs into genus 0 descendants, and the graph becomes precisely the graph for higher genus descendant potentials.

\section{REFERENCES}

[1] D. Abramovich and B. Fantechi, Orbifold techniques in degeneration formulas, arXiv: 1103.5132

[2] D. Abramovich, T. Graber, and A. Vistoli, Algebraic orbifold quantum products, Orbifolds in mathematics and physics (Madison, WI, 2001), 1-24, Contemp. Math. 310, Amer. Math. Soc., Providence, RI, 2002.

[3] D. Abramovich, T. Graber, and A. Vistoli, Gromov-Witten theory of Deligne-Mumford stacks, Amer. J. Math. 130 (2008), no. 5, 1337-1398.

[4] M. Aganagic, A. Klemm, M. Mariño, and C. Vafa, The topological vertex, Comm. Math. Phys. 254 (2005), no. 2, 425-478.

[5] M. Aganagic, A. Klemm, and C. Vafa, Disk instantons, mirror symmetry and the duality web, Z. Naturforsch. A 57 (2002), 1-28.

[6] M. Aganagic, M. Mariño, and C. Vafa, All loop topological string amplitudes from ChernSimons theory, Comm. Math. Phys. 247 (2004), no. 2, 467-512.

[7] M. Aganagic and C. Vafa, Mirror symmetry, D-branes and counting holomorphic discs, arXiv:hep-th/0012041.

\footnotetext{
${ }^{1}$ Iritani 68] does not explicitly states this identity under the SYZ transform, but instead he matches the cases $\mathcal{F}=\mathcal{O}_{\mathcal{X}}$ and a skyscraper sheaf. He then applies the monodromy to $\mathcal{O}_{\mathcal{X}}$ to obtain other line bundles on $\mathcal{X}$. These sheaves generate the K-theory group.
} 
[8] M. Alim and J. D. Länge, Polynomial structure of the (open) topological string partition function, J. High Energy Phys. 2007, no. 10, 045, 13pp.

[9] D. Arinkin and A. Polishchuk, Fukaya category and Fourier transform, Winter School on Mirror Symmetry, Vector Bundles and Lagrangian Submanifolds, AMS/IP Stud. Adv. Math., Vol. 23, 2001, pp. 261-274.

[10] M. F. Atiyah and R. Bott, The moment map and equivariant cohomology, Topology 23 (1984), no. 1, 1-28.

[11] V. V. Batyrev and D. A. Cox, On the Hodge structure of projective hypersurfaces in toric varieties, Duke Math. J. 75 (1994), no. 2, 293-338.

[12] K. Behrend, Gromov-Witten invariants in algebraic geometry, Invent. Math. 127 (1997), no. 3, 601-617.

[13] K. Behrend and B. Fantechi, Intrinsic normal cone, Invent. Math. 128 (1997), no. 1, 45-88.

[14] K. Behrend and Y. Manin, Stacks of stable maps and Gromov-Witten invariants, Duke Math. J. 85 (1996), no. 1, 1-60.

[15] M. Bershadsky, S. Cecotti, H. Ooguri, and C. Vafa, Holomorphic anomalies in topological field theories, Nuclear Phys. B 405 (1993), no. 2-3, 279-304.

[16] M. Bershadsky, S. Cecotti, H. Ooguri, and C. Vafa, Kodaira-Spencer theory of gravity and exact results for quantum string amplitudes, Comm. Math. Phys. 165 (1994), no. 2, 311-427.

[17] M. Bershadsky and A. Radul, Conformal field theories with additional $\mathbb{Z}_{n}$ symmetry, Int. J. Mod. Phys. A 02, (1987), 165.

[18] L. Borisov, L. Chen, and G. Smith, The orbifold Chow ring of toric Deligne-Mumford stacks, J. Amer. Math. Soc. 18 (2005), no. 1, 193-215.

[19] V. Bouchard, A. Catuneanu, O. Marchal, and P. Sułkowski, The remodeling conjecture and the Faber-Pandharipande formula, Lett. Math. Phys. 103 (2013), 59-77.

[20] V. Bouchard, A. Klemm, M. Mariño, and S. Pasquetti, Remodeling the B-model, Comm. Math. Phys. 287 (2009), no. 1, 117-178.

[21] V. Bouchard, A. Klemm, M. Mariño, and S. Pasquetti, Topological open strings on orbifolds, Comm. Math. Phys. 296 (2010), no. 3, 589-623.

[22] A. Brini, Open topological strings and integrable hierarchies: Remodeling the A-model, Comm. Math. Phys. 312 (2012), no. 3, 735-780.

[23] J. Bryan, C. Cadman, and B. Young, The orbifold topological vertex, Adv. Math. 229 (2012), no. 1, 531-595.

[24] P. Candelas, X. C. de la Ossa, P. S. Green, and L. Parkes, A pair of Calabi-Yau manifolds as an exactly soluble superconformal theory, Nuclear Phys. B 359 (1991), no. 1, 21-74.

[25] S. Cecotti and C. Vafa, Massive orbifolds, Mod. Phys. Lett. A 07 (1992), 1715.

[26] K. Chan, C.-H. Cho, S.-C. Lau, and H.-H. Tseng, Gross fibrations, SYZ mirror symmetry, and open Gromov-Witten invariants for toric Calabi-Yau orbifolds, preprint, arXiv:1306.0437 J. Differential Geom. 103 (2016), no. 2, 207-288.

[27] K. Chan, S.-C. Lau, and N. C. Leung, SYZ mirror symmetry for toric Calabi-Yau manifolds, J. Differential Geom. 90 (2012), no. 2, 177-250.

[28] L. Chen, Bouchard-Klemm-Marino-Pasquetti conjecture for $\mathbb{C}^{3}$, arXiv:0910.3739

[29] B. Chen, A.-M. Li, S. Sun, and G. Zhao, Relative orbifold Gromov-Witten theory and degeneration formula, arXiv:1110.6803

[30] W. Chen and Y. Ruan, Orbifold Gromov-Witten theory, Orbifolds in mathematics and physics, 25-85, Contemp. Math. 310, Amer. Math. Soc., Providence, RI, 2002.

[31] W. Chen and Y. Ruan, A new cohomology theory of orbifold, Comm. Math. Phys. 248 (2004), no. 1, 131.

[32] T.-M. Chiang, A. Klemm, S.-T. Yau, and E. Zaslow, Local mirror symmetry: calculations and interpretations, Adv. Theor. Math. Phys. 3 (1999), 495-565.

[33] T. Coates, A. Corti, H. Iritani, and H.-H. Tseng, A mirror theorem for toric stacks, Compos. Math. 151 (2015), no. 10, 1878-1912.

[34] K. J. Costello, Renormalization and effective field theory, Mathematical Surveys and Monographs, Vol. 170, American Mathematical Society, Providence, RI, 2011.

[35] K. J. Costello and S. Li, Quantum BCOV theory on Calabi-Yau manifolds and the higher genus B-model, arXiv:1201.4501.

[36] D. A. Cox and S. Katz, Mirror symmetry and algebraic geometry, Mathematical Surveys and Monographs 68, American Mathematical Society, Providence, RI, 1999. 
[37] P. Deligne and D. Mumford, The irreducibility of the space of curves of given genus, Inst. Hautes Études Sci. Publ. Math. No. 36 (1969), 75-109.

[38] D. E. Diaconescu and B. Florea, Localization and gluing of topological amplitudes, Comm. Math. Phys. 257 (2005), 119-149.

[39] L. Dixon, D. Friedan, E. Martinec, and S. Shenker, The conformal field theory of orbifolds, Nucl. Phys. B 282 (1987), 13-73.

[40] L. Dixon, J. A. Harvey, C. Vafa, and E. Witten, Strings on orbifolds, Nuclear Phys. B 261 (1985), no. 4, 678-686.

[41] L. Dixon, J. A. Harvey, C. Vafa, and E. Witten, Strings on orbifolds II, Nuclear Phys. B 274 (1986), no. 2, 285-314.

[42] S. K. Donaldson and R. P. Thomas, Gauge theory in higher dimensions, The geometric universe (Oxford, 1996), 31-47, Oxford Univ. Press, Oxford, 1998.

[43] P. Dunin-Barkowski, N. Orantin, S. Shadrin, and L. Spitz, Identification of the Givental formula with the special curves topological recursion procedure, Comm. Math. Phys. 328 (2014), no. 2, 669-700.

[44] B. Eynard, Intersection numbers and spectral curves, arXiv:1104.0176

[45] B. Eynard, Invariants of spectral curves and intersection theory of moduli spaces of complex curves, Commun. Number Theory Phys. 8 (2014), no. 3, 541-588.

[46] B. Eynard and N. Orantin, Invariants of algebraic curves and topological expansion, Commun. Number Theory Phys. 1 (2007), no. 2, 347-452.

[47] E. Eynard and N. Orantin, Computation of open Gromov-Witten invariants for toric CalabiYau 3-folds by topological recursion, a proof of the BKMP conjecture, Comm. Math. Phys. 337 (2015), no. 2, 483-567.

[48] C. Faber and R. Pandharipande, Hodge integrals and Gromov-Witten theory, Invent. Math 139 (2000), no. 1, 173-199.

[49] B. Fang and C.-C. M. Liu, Open Gromov-Witten invariants of toric Calabi-Yau 3-folds, Comm. Math. Phys. 323 (2013), no. 1, 285-328.

[50] B. Fang, C.-C. M. Liu, and H.-H. Tseng, Open-closed Gromov-Witten invariants of 3dimensional Calabi-Yau smooth toric DM stacks, arXiv:1212.6073

[51] B. Fang, C.-C. M. Liu, and Z. Zong, All genus open-closed mirror symmetry for affine toric Calabi-Yau 3-orbifolds, arXiv:1310.4818

[52] B. Fang, C.-C. M. Liu, and Z. Zong, On the remodeling conjecture for toric Calabi-Yau 3-orbifolds, arXiv: 1604.07123

[53] J. D. Fay, Theta functions on Riemann surfaces, Lecture Notes in Mathematics, Vol. 352. Springer-Verlag, Berlin-New York, 1973.

[54] K. Fukaya and K. Ono, Arnold conjecture and Gromov-Witten invariant, Topology 38 (1999), no. 5, 933-1048.

[55] A. B. Givental, Equivariant Gromov-Witten invariants, Internat. Math. Res. Notices 1996, no. $13,613-663$.

[56] A. B. Givental, A mirror theorem for toric complete intersections, Topological field theory, primitive forms and related topics (Kyoto, 1996), 141-175, Progr. Math. 160, Birkhäuser Boston, Boston, MA, 1998.

[57] A. B. Givental, Semisimple Frobenius structures at higher genus, IMRN (2001), no. 23, $1265-1286$.

[58] A. B. Givental, Gromov-Witten invariants and quantization of quadratic hamiltonians, Mosc. Math. J. 1 (2001), no. 4, 551-568.

[59] A. B. Givental, Symplectic geometry of Frobenius structures, Frobenius manifold, 91-112, Aspects Math., E36, Friedr. Vieweg, Wiesbanden 2004.

[60] T. Graber and R. Pandharipande, Localization of virtual classes, Invent. Math. 135 (1999), no. $2,487-518$.

[61] T. Graber and E. Zaslow, Open-string Gromov-Witten invariants: calculations and a mirror 'theorem', Orbifolds in mathematics and physics (Madison, WI, 2001), 107-121, Contemp. Math. 310, Amer. Math. Soc., Providence, RI, 2002.

[62] M. Gross, L. Katzarkov, and H. Ruddat, Towards mirror symmetry for varieties of general type, arXiv:1202.4042

[63] S. Hamidil and C. Vafa, Interactions on orbifolds, Nuclear Physi. B 279 (1987), 465-513.

[64] R. Harvey and H. B. Lawson, Jr., Calibrated geometries, Acta Math. 148 (1982), 47-157.

[65] K. Hori, A. Iqbal, and C. Vafa, D-branes and mirror symmetry, arXiv: hep-th/0005247 
[66] K. Hori and C. Vafa, Mirror symmetry, arXiv:hep-th/0002222

[67] M.-X. Huang, A. Klemm, and S. Quackenbush, Topological String Theory on Compact Calabi-Yau: Modularity and Boundary Conditions, Homological mirror symmetry, 45-102, Lecture Notes in Phys. 757, Springer, Berlin, 2009.

[68] H. Iritani, An integral structure in quantum cohomology and mirror symmetry for toric orbifolds, Adv. Math. 222 (2009), no. 3, 1016-1079.

[69] T. J. Jarvis and T. Kimura, Orbifold quantum cohomology of the classifying space of a finite group, Orbifolds in mathematics and physics (Madison, WI, 2001), 123-134, Contemp. Math. 310, Amer. Math. Soc., Providence, RI, 2002.

[70] I. Kostov, N. Orantin, "CFT and topological recursion," J. High Energy Phys. 2010, no. $11,056,30 \mathrm{pp}$.

[71] J. Kaczorowski and A. Perelli, A uniform version of Stirling's formula, Funct. Approx. Comment. Math. 45 (2011), part 1, 89-96.

[72] S. Katz and C.-C. M. Liu, Enumerative geometry of stable maps with Lagrangian boundary conditions and multiple covers of the disc, Adv. Theor. Math. Phys. 5 (2001), no. 1, 1-49.

[73] A. Klemm and E. Zaslow, Local mirror symmetry at higher genus, Winter School on Mirror Symmetry, Vector Bundles and Lagrangian Submanifolds (Cambridge, MA, 1999), 183-207, AMS/IP Stud. Adv. Math. 23, Amer. Math. Soc., Providence, RI, 2001.

[74] V. G. Knizhnik, Analytic fields on Riemann surfaces. II, Comm. Math. Phys. 112, no. 4 (1987), 567-590.

[75] M. Kontsevich, Intersection theory on the moduli space of curves and the matrix Airy function, Comm. Math. Phys. 147 (1992), no. 1, 1-23.

[76] M. Kontsevich, Enumeration of rational curves via torus actions, The moduli space of curves (Texel Island, 1994), 335-368, Progr. Math. 129, Birkhäuser Boston, Boston, MA, 1995.

[77] Y. P. Lee and R. Pandharipande, Frobenius manifolds, Gromov-Witten theory and Virasoro constraints.

[78] N.-C. Leung, S.-T. Yau, and E. Zaslow, From special Lagrangian to Hermitian-Yang-Mills via Fourier-Mukai transform, Adv. Theor. Math. Phys. 4 (2000) 1319-1341.

[79] A. Li and Y. Ruan, Symplectic surgery and Gromov-Witten invariants of Calabi-Yau 3folds, Invent. Math. 145 (2001), no. 1, 151-218.

[80] J. Li, Stable Morphisms to singular schemes and relative stable morphisms, J. Diff. Geom. 57 (2001), 509-578.

[81] J. Li, A degeneration formula of Gromov-Witten invariants, J. Diff. Geom. 60 (2002), 199293.

[82] J. Li, Recent progress in GW-invariants of Calabi-Yau threefolds, Current developments in mathematics, 2007, 77-99, Int. Press, Somerville, MA, 2009.

[83] J. Li, C.-C. M. Liu, K. Liu, and J. Zhou, A mathematical theory of the topological vertex, Geom. Topol. 13 (2009), no. 1, 527-621.

[84] J. Li and G. Tian, Virtual moduli cycles and Gromov-Witten invariants of algebraic varieties, J. Amer. Math. Soc. 11 (1998), no. 1, 119-174.

[85] J. Li and G. Tian, Virtual moduli cycles and Gromov-Witten invariants of general symplectic manifolds, Topics in symplectic 4-manifolds (Irvine, CA, 1996), 47-83, First Int. Press Lect. Ser., I, Int. Press, Cambridge, MA, 1998.

[86] B. H. Lian, K. Liu, and S.-T. Yau, Mirror principle I, Asian J. Math. 1 (1997), no. 4, 729-763.

[87] B. H. Lian, K. Liu, and S.-T. Yau, Mirror principle III, Asian J. Math. 3 (1999), no. 4, $771-800$.

[88] C.-C. M. Liu, Moduli of J-holomorphic curves with Lagrangian boundary conditions and open Gromov-Witten invariants for an $S^{1}$-equivariant pair, arXiv:math/0211388.

[89] C.-C. M. Liu, Localization in Gromov-Witten theory and orbifold Gromov-Witten theory, Handbook of Moduli, Volume II, 353-425, Adv. Lect. Math., (ALM) 25, International Press and Higher Education Press, 2013.

[90] C.-C. M. Liu, K. Liu, and J. Zhou, A proof of a conjecture of Mariño-Vafa on Hodge Integrals, J. Differential Geom. 65 (2003), no. 2, 289-340.

[91] C.-C. M. Liu, K. Liu, and J. Zhou, A formula of two-partition Hodge integrals, J. Amer. Math. Soc. 20 (2007), no. 1, 149-184. 
[92] M. Mariño, Open string amplitudes and large order behavior in topological string theory, J. High Energy Phys. 2008, no. 3, 060, 34pp.

[93] D. Maulik, N. Nekrasov, A. Okounkov, and R. Pandharipande, Gromov-Witten theory and Donaldson-Thomas theory I, Compos. Math. 142 (2006), no. 5, 1263-1285.

[94] D. Maulik, N. Nekrasov, A. Okounkov, and R. Pandharipande, Gromov-Witten theory and Donaldson-Thomas theory II, Compos. Math. 142 (2006), no. 5, 1286-1304.

[95] D. Maulik, A. Oblomkov, A. Okounkov, and R. Pandharipande, Gromov-Witten/DonaldsonThomas correspondence for toric 3-folds, Invent. Math. 186 (2011), no. 2, 435-479.

[96] D. Maulik and R. Pandharipande, A topological view of Gromov-Witten theory, Topology 45 (2006), no. 5, 887-918.

[97] D. Mumford, Towards an enumerative geometry of the moduli space of curves, Arithmetic and geometry, Vol. II, 271-328, Progr. Math. 36, Birkhäuser Boston, Boston, MA, 1983.

[98] A. Okounkov and R. Pandharipande, Hodge integrals and invariants of the unknot, Geom. Topol. 8 (2004), 675-699.

[99] N. Orantin, "Symplectic invariants, Virasoro constraints and Givental decomposition," arXiv:0808.0635

[100] A. Popa, The genus one Gromov-Witten invariants of Calabi-Yau complete intersections, Trans. Amer. Math. Soc. 365 (2013), no. 3, 1149-1181.

[101] S.-S. Roan, On Calabi-Yau orbifolds in weighted projective spaces, Internat. J. Math. 1 (1990), no. 2, 211-232.

[102] D. Ross, Localization and gluing of orbifold amplitudes: the Gromov-Witten orbifold vertex, Trans. Amer. Math. Soc. 366 (2014), no. 3, 1587-1620.

[103] D. Ross, On the Gromov-Witten/Donaldson-Thomas correspondence and Ruan's conjecture for Calabi-Yau 3-orbifolds, Comm. Math. Phys. 340 (2015), no. 2, 851-864.

[104] D. Ross and Z. Zong, The Gerby Gopakumar-Mariño-Vafa Formula, Geom. Topol. 17 (2013), no. 5, 2935-2976.

[105] D. Ross and Z. Zong, Cyclic Hodge Integrals and Loop Schur Functions, Adv. Math. 285 (2015), 14481486. arXiv:1401.2217

[106] Y. Ruan, Virtual neighborhoods and pseudo-holomorphic curves, Proceedings of 6th Gökova Geometry-Topology Conference, Turkish J. Math. 23 (1999), no. 1, 161-231.

[107] Y. Ruan and G. Tian, A mathematical theory of quantum cohomology, J. Differential Geom. 42 (1995), no. 2, 259-367.

[108] Y. Ruan and G. Tian, Higher genus symplectic invariants and sigma models coupled with gravity, Invent. Math. 130 (1997), no. 3, 455-516.

[109] B. Siebert, Gromov-Witten invariants of general symplectic manifolds, arXiv: dg-ga/9608005

[110] A. Strominger, S.-T. Yau, and E. Zaslow, Mirror symmetry is T-duality, Nuclear Physics B 479 (1), 243-259.

[111] R. P. Thomas, A holomorphic Casson invariant for Calabi-Yau 3-folds, and bundles on K3 fibrations, J. Differential Geom. 54 (2000), no. 2, 367-438.

[112] H.-H. Tseng, Orbifold quantum Riemann-Roch, Lefschetz and Serre, Geom. Topol. 14 (2010), no. 1, 1-81.

[113] E. Witten, Two-dimensional gravity and intersection theory on moduli space, Surveys in differential geometry (Cambridge, MA, 1990), 243-310, Lehigh Univ., Bethlehem, PA, 1991.

[114] S. Yamaguchi, S.-T. Yau, Topological string partition functions as polynomials, J. High Energy Phys. 2004, no. 7, 047, 20pp.

[115] E. Zaslow, Topological orbifold models and quantum cohomology rings, Comm. Math. Phys. 156 (1993), no. 2, 301-331.

[116] J. Zhou, Local mirror symmetry for one-legged topological vertex, arXiv:0910.4320 Local Mirror symmetry for the topological vertex, arXiv:0911.2343

[117] J. Zhou, Open string invariants and mirror curve of the resolved conifold, arXiv:1001.0447.

[118] A. Zinger, The reduced genus 1 Gromov-Witten invariants of Calabi-Yau hypersurfaces, J. Amer. Math. Soc. 22 (2009), no. 3, 691-737.

[119] Z. Zong, Generalized Mariño-Vafa Formula and Local Gromov-Witten Theory of Orbicurves, J. Differential Geom. 100 (2015), no. 1, 161-190.

[120] Z. Zong, Equivariant Gromov-Witten theory of GKM orbifolds, arXiv:1604.07270 
Bohan Fang, Beijing International Center for Mathematical Research, Peking University, 5 Yiheyuan Road, Beijing 100871, China

E-mail address: bohanfang@gmail.com

Chiu-Chu Melissa Liu, Department of Mathematics, Columbia University, 2990 BroadWAY, NEW YORK, NY 10027, USA

E-mail address: ccliu@math.columbia.edu

Zhengyu Zong, Yau Mathematical Sciences Center, Tsinghua University, Jin Chun Yuan West Build., Tsinghua University, Beijing 100084, China

E-mail address: zyzong@math.tsinghua.edu.cn 